\title{
A hyperboloidal study of tail decay rates for scalar and Yang-Mills fields
}

\author{
Anıl Zenginoğlu \\ Max-Planck-Institut für Gravitationsphysik, Albert-Einstein-Institut, Am Mühlenberg 1, \\ D-14476 Golm, Germany \\ E-mail: anil@aei.mpg.de
}

Received 14 March 2008

Published 19 August 2008

Online at stacks.iop.org/CQG/25/175013

\begin{abstract}
We investigate the asymptotic behavior of spherically symmetric solutions to scalar wave and Yang-Mills equations on a Schwarzschild background. The studies demonstrate the astrophysical relevance of null infinity in predicting radiation signals for gravitational wave detectors and show how test fields on unbounded domains in black hole spacetimes can be simulated conveniently by numerically solving hyperboloidal initial value problems.
\end{abstract}

PACS numbers: 04.25.Dm, 03.65.Pm, 04.30.-w

\section{Introduction}

The purpose of this paper is two-fold. First, we emphasize the astrophysical relevance of null infinity in predicting gravitational radiation signals as they are expected to be detected by gravitational wave detectors. It has been demonstrated in [40] that a suitable feature to estimate the validity of the concept of null infinity when predicting detector signals is the tail behavior. In this paper, we work out this idea further by discussing the observer dependence of tails for solutions to scalar wave and Yang-Mills equations on a Schwarzschild background. Secondly, it is demonstrated that test fields at null infinity can be conveniently studied numerically by solving Cauchy problems once a suitable choice of gauge for the background spacetime has been made.

The question about the astrophysical relevance of null infinity is a conceptual one. Mathematically, it is only at null infinity that one can give an unambiguous definition of gravitational radiation. Many physicists, however, are uncomfortable with the notion of null infinity because the detectors are at a finite distance away from the source and move along timelike curves while idealized observers at null infinity are infinitely far away from the source and move along null curves. These issues are addressed within the paper on concrete examples. 
Our aim is not to discuss the gauge dependence of radiation extraction or the influence of finite distance boundary conditions on the numerical solution as has been done for example in $[35,41]$. We will rather numerically study a genuinely physical phenomenon, namely the crucial dependence of a certain feature in the radiation signal on the observer's distance from the source $[24,40]$.

There is compelling evidence suggesting that, asymptotically with respect to an appropriate time coordinate, the gravitational radiation flux from a perturbed black hole decays polynomially in time [13, 15, 16, 25, 26, 39]. This polynomial decay can be regarded as being due to back-scatter of gravitational radiation off the curved background spacetime. The back-scattering effect is very small but it may, in principle, be detected by future generation gravitational wave detectors [6]. The decay rate of the tail may be of particular interest in testing fundamental predictions of general relativity because uniqueness theorems suggest that all features of black holes other than mass, charge and angular momentum will decay according to this rate independent of the details of the collapse process. The tail plays an important role also in the mathematical discussion of stability of black hole spacetimes and the cosmic censorship conjecture $[15,16]$.

It turns out that the gravitational radiation flux of a general perturbation has a faster decay on the event horizon and on timelike surfaces than on null infinity [2, 3, 7, 10, 16, 25, 39]. This difference raises the question of which decay is the relevant one with respect to our gravitational wave detectors. Pürrer et al have demonstrated in [40] that the relevant decay rate in the so-called astrophysical zone [32] is the one measured at null infinity. Their study is based on a characteristic approach and discusses critical collapse for self-gravitating massless scalar fields in spherical symmetry.

We are interested in a detailed study of the observer dependence of decay rates including null infinity and assume the background to be given. We compare a powerful mathematical estimate on decay rates for scalar fields suggested in [42, 43] with numerical calculations and check decay rates for scalar fields with non-vanishing angular momentum. We also study the tail behavior for Yang-Mills fields where a breakdown of linear perturbation theory has been demonstrated at future timelike infinity [5]. Our study indicates a similar breakdown of the linear analysis along future null infinity.

Additionally, we show how test fields on black hole spacetimes can be studied numerically by including null infinity while solving Cauchy problems. Accurate studies of fields on black hole spacetimes including null infinity have been restricted to the characteristic approach until now [12, 25, 31]. The characteristic approach, however, has certain difficulties mainly due to problems related to the rigidity in the choice of the underlying gauge [44]. The method presented in this paper is quite flexible and allows us to use standard discretization techniques commonly applied in numerical relativity. The feature that makes the accuracy of our calculations possible is that the numerical outer boundary coincides with null infinity so that, in the continuum problem, no boundary conditions are required and no resolution loss appears in the physical part of the conformal extension [1, 17, 30, 46].

\section{The conformal method}

We want to study solutions to the scalar wave equation

$$
\tilde{\square} \tilde{\Phi}=0,
$$

for a scalar field $\tilde{\Phi}$ in a Schwarzschild spacetime. To include null infinity in the computational domain in a manifestly regular fashion, we apply the conformal method introduced by Penrose 
[36-38] (see also [19, 21, 28, 29]). A conformal rescaling $g=\Omega^{2} \tilde{g}$ of the physical metric $\tilde{g}$ with a conformal factor $\Omega>0$ implies the transformation

$$
\left(\square-\frac{1}{6} R\right) \Phi=\Omega^{-3}\left(\tilde{\square}-\frac{1}{6} \tilde{R}\right) \tilde{\Phi}, \quad \text { with } \quad \Phi=\frac{\tilde{\Phi}}{\Omega},
$$

where $R$ and $\tilde{R}$ are the Ricci scalars of the rescaled and the physical metrics $g$ and $\tilde{g}$ respectively. Equation (1) with respect to the rescaled metric then becomes

$$
\square \Phi-\frac{1}{6} R \Phi=0 .
$$

We are also interested in solutions to the Yang-Mills equations,

$$
D * F:=d * F+A \wedge F=0,
$$

where $F=d A+A \wedge A$ is the Yang-Mills curvature and $A$ is the Yang-Mills connection. The Yang-Mills equations are conformally invariant. We can therefore study the system (3) directly in a conformally rescaled spacetime.

Schwarzschild spacetime is weakly asymptotically simple, implying that a suitably rescaled metric is smoothly extendable through null infinity. We can solve the equations (2) and (3) in a conformally extended Schwarzschild spacetime by allowing the conformal factor to vanish in a certain way provided that the initial data have sufficiently fast fall-off [23].

To solve an initial value problem for (2) and (3) numerically on a Schwarzschild spacetime, we need to choose a coordinate system that is suitable for numerical calculations and covers that part of the Schwarzschild-Kruskal manifold we are interested in, namely the domain extending from a neighborhood of the event horizon up to future null infinity. Our coordinates should also be adapted to a natural family of observers, each being thought of as representing gravitational wave detectors at constant distances away from the black hole.

A convenient foliation covering the extended Schwarzschild spacetime up to future null infinity satisfying the above requirements can be found in the class of spherically symmetric constant mean curvature (CMC) foliations of Schwarzschild spacetime [9, 22, 33]. The transformation from the standard Schwarzschild time coordinate to the time coordinate of a spherically symmetric CMC foliation can be written as

$$
t=\tilde{t}-h(\tilde{r}),
$$

where $\tilde{r}$ is the standard Schwarzschild area radius, $h(\tilde{r})$ is the height function and $\tilde{t}$ is the standard Schwarzschild time coordinate. The derivative of the height function is given by

$$
h^{\prime}(\tilde{r})=\frac{\frac{\tilde{K} \tilde{r}^{3}}{3}-C}{\left(1-\frac{2 m}{\tilde{r}}\right) \tilde{P}(\tilde{r})}
$$

with

$$
\widetilde{P}(\tilde{r}):=\sqrt{\left(\frac{\tilde{K} \tilde{r}^{3}}{3}-C\right)^{2}+\left(1-\frac{2 m}{\tilde{r}}\right) \tilde{r}^{4}} .
$$

The mass of the Schwarzschild black hole is denoted by $m$. The foliation parameters are the mean extrinsic curvature $\tilde{K}$, and a constant of integration $C$. The global behavior of CMCsurfaces depends on the foliation parameters. We choose the parameters such that the surfaces of the foliation come from future null infinity, pass the event horizon above the bifurcation sphere and run into the future singularity as depicted in figure 1.

The transformation (4) implies that the coordinates of the foliation are adapted to time symmetry in the sense that the timelike Killing vector field is given by $\partial_{t}$. To keep this 


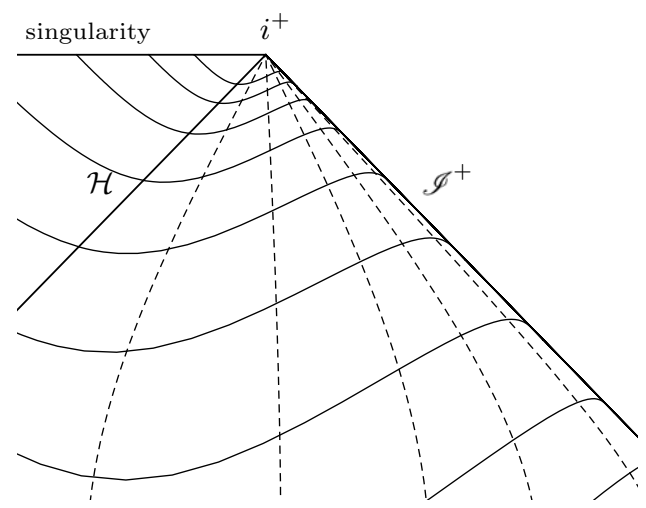

Figure 1. Penrose diagram of a CMC foliation in Schwarzschild spacetime with $m=1 / 2, C=$ 1, $\tilde{K}=2$. The dashed lines represent Killing observers.

property for the conformally rescaled metric, we perform the conformal compactification such that the conformal factor is time-independent which we refer to as conformal fixing. The Schwarzschild metric in a CMC foliation and a conformal fixing gauge can be written as [46]

$g=-\left(1-\frac{2 m \Omega}{r}\right) \Omega^{2} \mathrm{~d} t^{2}-\frac{2\left(\tilde{K} r^{3} / 3-C \Omega^{3}\right)}{P(r)} \mathrm{d} t \mathrm{~d} r++\frac{r^{4}}{P^{2}(r)} \mathrm{d} r^{2}+r^{2} \mathrm{~d} \sigma^{2}$,

where $\mathrm{d} \sigma^{2}$ is the standard metric on the unit sphere,

$$
P(r):=\sqrt{\left(\frac{\tilde{K} r^{3}}{3}-C \Omega^{3}\right)^{2}+\left(1-\frac{2 m(1-r)}{r}\right) \Omega^{2} r^{4}}
$$

and $\Omega=1-r$. Note that conformal fixing on a hyperboloidal foliation implies scri-fixing, that is, the coordinate location of null infinity in a conformal fixing gauge is independent of the time coordinate. The compactifying coordinate $r$ is related to the standard Schwarzschild area-radius $\tilde{r}$ via

$$
\tilde{r}=\frac{r}{1-r}=\frac{r}{\Omega} .
$$

The timelike curves with constant spatial coordinates can be regarded as worldlines of natural observers at constant distances away from the source. As can be seen from figure 1 and from $g\left(\partial_{t}, \partial_{t}\right){\mid \mathscr{I}^{+}}=0$, the worldline of such observers becomes null at null infinity.

\section{The initial value problem}

\subsection{The continuum problem}

We write the metric (6) as

$$
g=\left(-\alpha^{2}+\gamma^{2} \beta^{2}\right) \mathrm{d} t^{2}+2 \gamma^{2} \beta \mathrm{d} t \mathrm{~d} r+\gamma^{2} \mathrm{~d} r^{2}+r^{2} \mathrm{~d} \sigma^{2} .
$$

The lapse $\alpha$, the shift $\beta$ and the spatial metric function $\gamma$ read

$$
\alpha=\frac{P(r)}{r^{2}}, \quad \beta=\left(-\frac{\tilde{K} r}{3}+\frac{C \Omega^{3}}{r^{2}}\right) \alpha, \quad \gamma=\frac{1}{\alpha} .
$$


We apply the method of separation of variables to (2). We write $\Phi(t, r, \vartheta, \varphi)=$ $\phi(t, r) Y_{l m}(\vartheta, \varphi)$ with $Y_{l m}$ being the usual spherical harmonics and define the auxiliary variables

$$
\psi:=\partial_{r} \phi \quad \text { and } \quad \pi:=\frac{\gamma}{\alpha}\left(\partial_{t} \phi-\beta \partial_{r} \phi\right) .
$$

Then the following linear, symmetric hyperbolic system of evolution equations for $\phi(t, r)$ is obtained as

$$
\begin{aligned}
& \partial_{t} \phi=\frac{\alpha}{\gamma} \pi+\beta \psi, \quad \partial_{t} \psi=\partial_{r}\left(\frac{\alpha}{\gamma} \pi+\beta \psi\right), \\
& \partial_{t} \pi=\frac{1}{r^{2}} \partial_{r}\left(r^{2}\left(\frac{\alpha}{\gamma} \psi+\beta \pi\right)\right)-\alpha \gamma\left(\frac{1}{6} R \phi-\frac{l(l+1)}{r^{2}} \phi\right) .
\end{aligned}
$$

The Ricci scalar of the metric (6) reads

$$
R=\frac{12 \Omega}{r^{2}}(r+m(2 r-1))
$$

For the Yang-Mills equations (3) we consider the gauge group $S U$ (2) and make the following spherically symmetric ansatz for the connection $[4,14]$ :

$$
A=(\phi+1) \sigma_{1} \mathrm{~d} \vartheta+\left(\cos \vartheta \sigma_{3}+(\phi+1) \sin \vartheta \sigma_{2}\right) \mathrm{d} \varphi,
$$

where $\sigma_{i}$ are Pauli matrices and $\phi=\phi(t, r)$. With the above choice, a vacuum state for the Yang-Mills field is given by $\phi=0$. The auxiliary variables are defined as in (9). The only difference to the system (10) is the equation for the time derivative of $\pi$ which becomes

$$
\partial_{t} \pi=\partial_{r}\left(\frac{\alpha}{\gamma} \psi+\beta \pi\right)-\frac{\alpha \gamma}{r^{2}} \phi(1+\phi)(2+\phi) .
$$

We choose an approximately outgoing, compactly supported Gaussian pulse for initial data. On the initial hypersurface such data satisfy

$$
\partial_{t}(r \phi)+c_{+} \partial_{r}(r \phi)=0
$$

where $c_{+}$is the outgoing characteristic speed given by $c_{+}=-\beta+\alpha / \gamma$. The data read for $r<r_{\text {out }}=$ const $<1$ as follows,

$$
\begin{aligned}
& \phi(0, r)=a \mathrm{e}^{-\left(r-r_{c}\right)^{2} / \sigma^{2}}, \quad \psi(0, r)=-\frac{2\left(r-r_{c}\right)}{\sigma^{2}} \phi(0, r), \\
& \pi(0, r)=-\psi(0, r)-\frac{\phi(0, r)}{r}\left(1-\frac{\beta \gamma}{\alpha}\right),
\end{aligned}
$$

where $r_{c}$ is the center of the Gaussian pulse, $a$ is its amplitude and $\sigma$ is its width. The data are set to zero for $r \geqslant r_{\text {out }}$.

The initial hypersurface is hyperboloidal, meaning that it is a smooth spacelike hypersurface extending through null infinity. The problem consisting of initial data given on a hyperboloidal surface and evolution equations is called a hyperboloidal initial value problem [20]. Note that no boundary conditions are needed in the continuum problem.

\subsection{The numerical method}

We may solve hyperboloidal initial value problems for scalar wave and Yang-Mills equations using standard numerical techniques. I used the method of lines with fourth order Runge-Kutta time integration and finite differencing with sixth order accurate stencils. The inner boundary is chosen to be a spacelike surface inside the event horizon so that one can use an excision technique. The outer boundary is at null infinity where one-sided finite differencing is applied. 


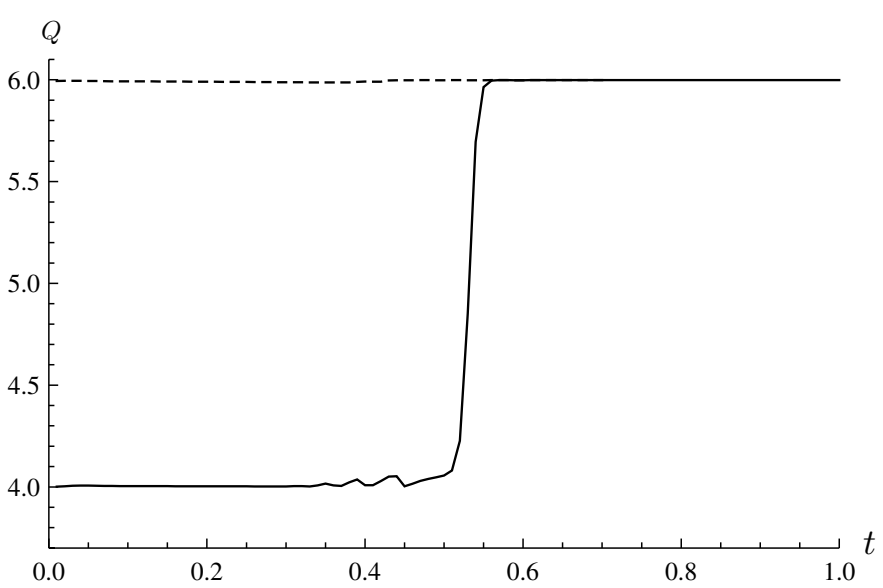

Figure 2. Convergence in the L2-norm for numerical solutions to (10). The convergence factor $Q$ is calculated by $Q=\log _{2} \frac{\left\|\phi^{\text {low }}-\phi^{\text {med }}\right\|}{\left\|\phi^{\text {med }}-\phi^{\text {high }}\right\|}$.

The lack of outer boundary conditions makes the numerical implementation simpler than in the case where an artificial timelike outer boundary is introduced.

The convergence of the code can be seen in figure 2 . For this plot, a three-level convergence analysis has been performed with 1010, 2020 and 4040 grid cells. The Courant factor $\Delta t / \Delta r$ is chosen to be 0.5 . The convergence in the L2-norm has been plotted for a solution to the hyperboloidal initial value problem for the scalar wave equation. The corresponding plot for the Yang-Mills system is similar. We see that the code switches from fourth- to sixth-order convergence after a short time. This seems to be due to the initially dominating error from the fourth-order time integration, and indeed, choosing a Courant factor 10 times smaller results in the dashed curve which indicates sixth-order convergence from the start. As we are interested in long time evolutions, the larger Courant factor is chosen. The convergence factors in the maximum and the L1-norms behave similarly.

For the results presented below the number of grid cells is chosen to be 10020 . The spatial simulation domain in grid coordinates is given by $r \in[0.499,1]$. The Courant factor is 0.2 . The parameters of the spacetime and the foliation are $m=0.5, \tilde{K}=0.5, C=1$.

\section{Results}

\subsection{Tails for the scalar wave equation}

The late-time decay of a solution to the scalar wave equation is expected to have the form

$$
\lim _{t \rightarrow \infty} \phi(r, t)=C t^{p}
$$

where $C$ depends on $r$ only and $p$ is the decay rate with $p<0$. For a solution with vanishing angular momentum, that is, $l=0$, we expect along the event horizon and along timelike surfaces a decay rate $p=-3$ [39]. At null infinity, however, the decay rate is expected to be $p=-2[7,25]$. We would like to know which decay rate is the relevant one in the context of astrophysical predictions and have a quantitative understanding of the dependence of the decay rate on the observer's location [40]. 


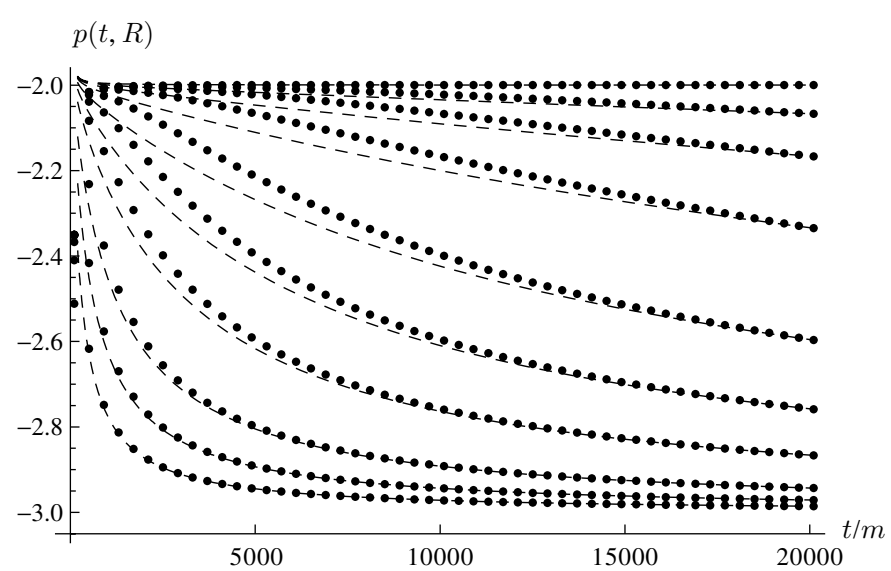

Figure 3. Comparison between the mathematical pointwise estimate (13) and a numerical calculation. Each curve depicts the function $p(t, R)$ calculated as in (14) for the following values $R / m=(\infty, 40000,20000,10000,4000,2000,1000,400,200,100)$.

4.1.1. Comparison between mathematical and numerical results. Recently, an optimal pointwise decay estimate has been suggested, but not yet proven, for small spherically symmetric solutions to nonlinear wave equations with a potential [42, 43]. Applying this estimate to our linear problem gives

$$
|\tilde{\phi}| \leqslant \frac{C_{1}}{\left(C_{2}+\tilde{t}+\tilde{r}_{*}\right)\left(C_{3}+\tilde{t}-\tilde{r}_{*}\right)^{2}},
$$

where $\left(C_{1}, C_{2}, C_{3}\right)$ are constants, $\tilde{r}_{*}:=\tilde{r}+2 m \log (\tilde{r}-2 m)$ and $\tilde{t}$ is the standard Schwarzschild time coordinate. This estimate captures the asymptotic behavior of the solution near null infinity as well as at finite distances from the black hole. The corresponding decay rates can easily be read off from (13). We want to compare the mathematical estimate with the numerical calculation with respect to the prediction of the decay rate. We calculate the function

$$
p(t, \tilde{r})=\frac{d \ln |\phi(t, \tilde{r})|}{d \ln t},
$$

which becomes asymptotically the exponent of the polynomial decay of the solution. For the comparison, we need to write the mathematical estimate (13) in the coordinates of our numerical calculation. The coordinate transformation is given by (4). The height function in this transformation is not given in explicit form, but as we are mainly interested in the asymptotic behavior of the solution in the far-field zone we can use a Taylor expansion of (5) as $\tilde{r} \rightarrow \infty$. We get up to a constant

$$
h(\tilde{r})=\tilde{r}_{*}+\frac{1}{\tilde{r}}\left(\frac{9}{2 K^{2}}-4 m^{2}\right)-\frac{4 m^{3}}{\tilde{r}^{2}}+O\left(\frac{1}{\tilde{r}^{3}}\right) .
$$

The comparison has been plotted in figure 3. Each curve in the figure gives the function $p(t, R)$ for a constant value of $R$. The dashed lines represent the mathematical estimates which have been fitted at late times to some representative data points from the numerical simulation. We set $C_{1}=C_{2}=1$ and treat $C_{3}$ as a fitting parameter along each curve. Considering that formula (13) is only an estimate and not an approximation to the solution, we can say that it is remarkable how well the qualitative behavior of the asymptotic decay is captured by it. 
4.1.2. Astrophysical relevance of null infinity for the decay rate. Now we can discuss the question of which decay rate is the relevant one for gravitational wave detectors. We think of each curve in figure 3 as representing the measurement made by a detector at some constant distance away from the source. In a Schwarzschild spacetime we have a length scale at our disposal given by $m$, the mass of the black hole, so that we can gain an intuition for the scales by considering the distances of some typical astronomical object in terms of geometric units. The closest candidate for a supermassive black hole is $\operatorname{Sgr} A^{*}$ at the center of our galaxy. Its mass is about $3.7 \times 10^{6} M_{\odot}$ and it is about 26000 light years away. In geometric units, this corresponds to roughly $10^{10} \mathrm{~m}$. Another close example is the first serious candidate for a stellar-size black hole, namely the compact object in the binary system Cygnus X-1. The total mass of the binary system is about $50 M_{\odot}$ and its distance to the Earth is about 8000 light years. This gives in geometric units a distance of roughly $10^{15} \mathrm{~m}$. Now consider in figure 3 the curve that is closest to what has been calculated along $\mathscr{I}^{+}$. Its distance to the black hole in geometric units is $4 \times 10^{4} \mathrm{~m}$. While this is quite far for numerical calculations, it is obviously still very close compared to astronomical distances. Even for such comparably small distances we see that the relevant decay rate is the one measured at null infinity.

One can of course argue that the decay rate along any timelike surface at a finite distance will eventually approach -3 if one waits long enough. It is clear from figure 3 and from the estimate (13) that the time scale for this to happen depends on the distance of the source. If we have a source that is, for example, 1000 light years away from the Earth, then the decay rate will come close to -3 only after about 10000 years. This is in accordance with the argument that the mathematical idealization of the region $\tilde{r} \gg \tilde{t}$ for radiative properties of fields corresponds to null infinity while for $\tilde{t} \gg \tilde{r}_{*}$ the correct idealization is timelike infinity [18, 32, 40]. Figure 3 depicts the transition zone between these two regions. We should mention that it is extremely unlikely that the polynomially weakening tail signal can be followed for such a long time that the decay rate at timelike infinity will be relevant. It is rather likely that if ever the tail decay rate is measured by direct observation, the measurement will be possible only for a short time.

The above discussion recapitulates that $\mathscr{I}^{+}$is the appropriate idealization for an observer's location in an asymptotically flat spacetime. One should, however, be aware that future generation detectors will observe variations in the radiation signal due to cosmological effects when the sources are considerably far away in comparison to the size of the known universe. In this case, one might reconsider the relationship between the different idealizations of isolated systems and cosmological spacetimes.

4.1.3. Construction of a physically motivated time coordinate. In our discussions and conclusions we use the coordinate time $t$. In this subsection we want to briefly elaborate that the same conclusions can be drawn with respect to a physically motivated time coordinate.

We have chosen our foliation suitably such that worldlines of natural observers of gravitational radiation are represented by timelike coordinate lines. The parametrization of these curves by $t$, however, can be regarded as being arbitrary. There are two steps to be taken in the construction of a more physically motivated time coordinate. First, we can synchronize our observers by an outgoing light pulse so that they have a common starting time for their measurements. This starting time $t_{R}$ for an observer located at $\tilde{r}=R$ can be calculated by

$$
\int_{t_{R}}^{T} \mathrm{~d} t=T-t_{R}=\int_{R}^{\infty} c_{+}(\tilde{r}) \mathrm{d} \tilde{r},
$$

where $T$ is the time at infinity and $c_{+}$is the outgoing speed of light rays. It does not matter whether $c_{+}$is calculated with respect to the physical or the conformally rescaled metric because 


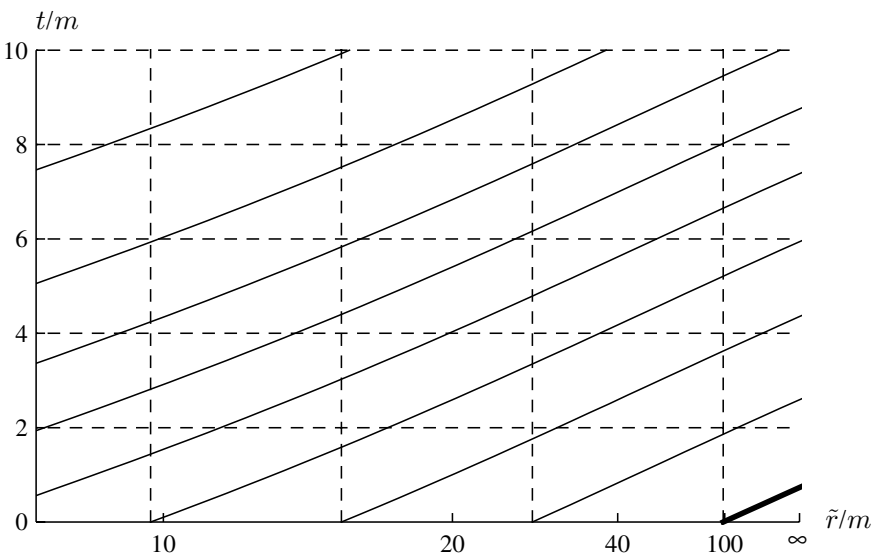

Figure 4. Grid diagram depicting outgoing null curves on the grid. To synchronize observers from $100 \mathrm{~m}$ to infinity, we need to take care of the shift in time given by the height of the thick curve. The diagram shows that the required shift is negligible in comparison to the time scales we are interested in.

the null cone structure is invariant under conformal rescalings. We depict in figure 4 null curves on our grid. We see that the difference in the initial time shift required for the synchronization is almost negligible for observers sufficiently far away. The reason for this is the fact that surfaces of a future hyperboloidal foliation follow closely outgoing null surfaces. Note that in a Cauchy-type foliation, the synchronizing shift in time would be calculated with respect to a far away observer at a finite distance. The required shift would then be on the order of the distance of that observer.

The next step for the construction of a physically motivated time parameter would be the reparametrization of the time coordinate so that the measurement by a given observer is plotted in proper time. The relation of the proper time of a Killing observer at $\tilde{r}=R$ to the coordinate time is given by

$$
\triangle s=\int \sqrt{-\tilde{g}_{t t}} \mathrm{~d} t=\left(1-\frac{2 m}{R}\right)^{1 / 2} \Delta t .
$$

We see that at infinity proper time corresponds to coordinate time. The rescaling makes only a small difference for observers far away.

A transformation of the results presented in this paper according to the choice of such a physically motivated time coordinate does not change the conclusions for far away observers. Therefore, and because of simplicity, the plots will be given in the local time coordinate of the numerical solution. Note, however, that we do not plot the decay rates for observers near the horizon in our diagrams although our coordinates do allow this as well.

4.1.4. A remark on finite distance extractions. In numerical calculations where an artificial timelike outer boundary is introduced, one extracts gravitational radiation information from the numerical solution at finite distances away from the source. When the extracted information does not vary in an unexpected manner with extraction radius or algorithm, one suggests that the calculation delivers a reliable answer. This practice can be regarded as safe as long as one has a theoretical understanding of the expected radiation signal which delivers tests of the result, such as the peeling behavior, conservation of mass or waveforms from approximation 


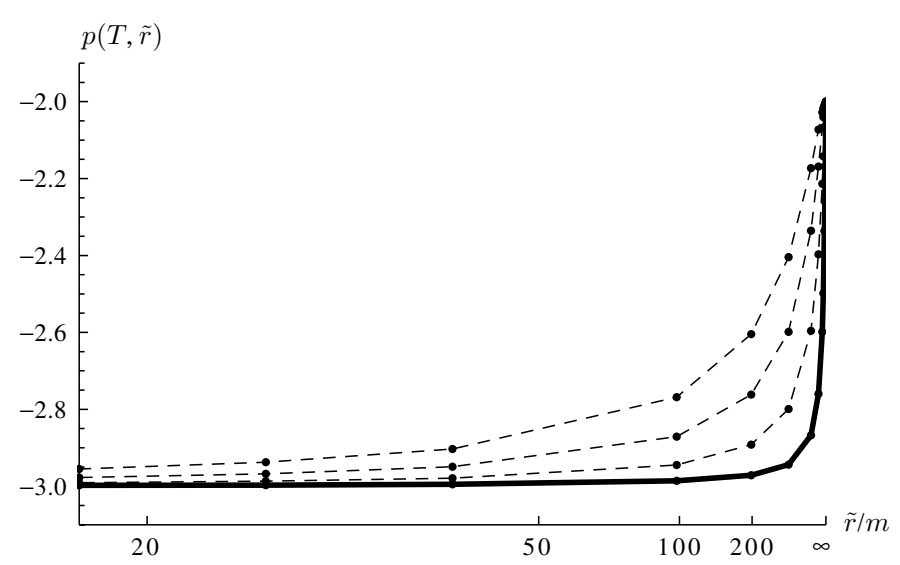

Figure 5. Observer dependence of the tail decay rates at subsequent times. The thick curve corresponds to $T=20000 \mathrm{~m}$.

methods to compare with [8, 27]. A naive interpretation of finite distance extractions without theoretical knowledge, however, can be misleading in terms of results as well as error estimates.

The decay rates at late times provide an example for a case which demands caution. In figure 5 we present a different way of looking at the same data as given in figure 3 . Here, decay rates at different finite distances have been plotted on subsequent time steps. The thick curve corresponds to the observer dependence of the decay rates at late times. We see that a direct Richardson extrapolation based on finite distance decay rates would not only deliver a wrong result, but also a wrong error estimate.

4.1.5. Scalar field with angular momentum. The difference in the expected tail behavior at null infinity and finite distances becomes larger with $l$. At finite distances one expects the exponent to be $p=-2 l-3$, whereas at null infinity $p=-l-2$. In figure 6 we plot the decay rates for $l=1$ after the quasinormal mode ringing is over and the tail part of the solution remains. In this case, there is no conjecture of an optimal pointwise decay estimate yet available in the literature, so we only plot the result of the numerical calculation.

The qualitative behavior depicted in figure 6 is similar to that depicted in figure 3 which suggests that an estimate similar to (13) with modified exponents can be expected also for higher multipoles.

\subsection{Tails for the Yang-Mills equation}

The conformal method can be applied to study other fields as well. The basic requirements are that the equations transform in a well-defined manner under conformal rescalings of the metric and the fields have suitable asymptotic behavior. Yang-Mills equations are conformally invariant so that we can study them directly with the conformal method.

The tail behavior for Yang-Mills equations has been studied in [11] using linear perturbation analysis. They predict $p=-5$ at future timelike infinity and $p=-3$ at future null infinity. Bizon et al showed in [5] that the linear perturbation theory applied in [11] does not capture the correct tail behavior for Yang-Mills equations where nonlinear terms appear (11). Instead they argued and numerically demonstrated that the tail exponent at timelike infinity is $p=-4$ both for Minkowski and for Schwarzschild spacetimes. 


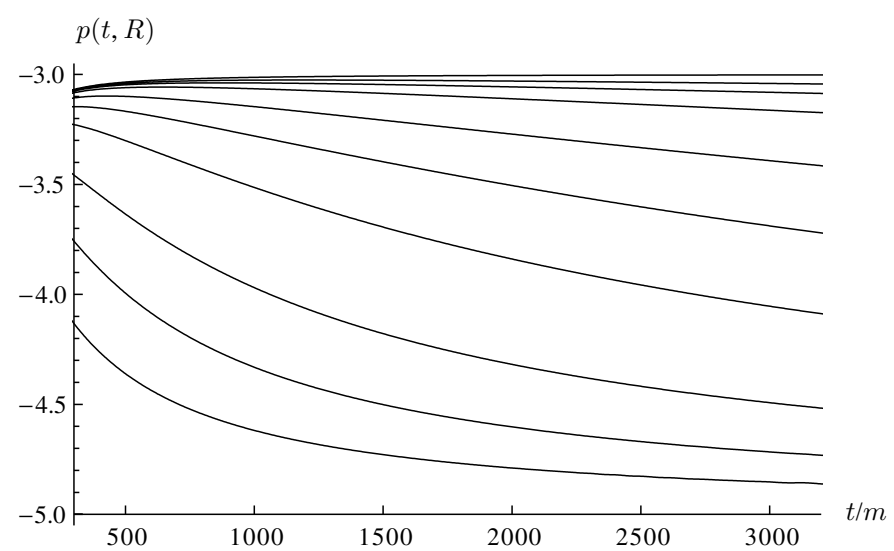

Figure 6. Decay rates for a solution to the scalar wave equation on a Schwarzschild background with $l=1$ plotted for the same observers as for figure 3 . This plot covers a shorter time than figure 3 due to the faster fall-off of the field.

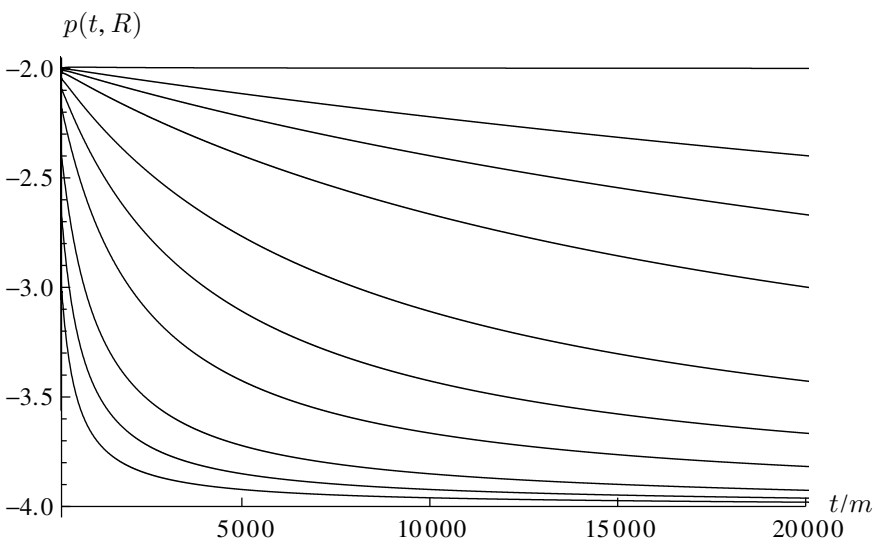

Figure 7. Decay rates for a solution to Yang-Mills equations on a Schwarzschild background. Each curve depicts the function $p(t, R)$ calculated as in (14) for the following values $R / m=$ $(\infty, 40000,20000,10000,4000,2000,1000,400,200,100)$.

We plot in figure 7 the observer dependence of decay rates for a solution of the YangMills equations. Near future timelike infinity at a decay rate of $p=-4$ is observed in accordance with [5]. One might expect that the prediction of [11] at future null infinity will also be wrong at the same rate which indeed seems to be the case. As seen in figure 7, the numerical calculation suggests a decay rate of $p=-2$ for Yang-Mills fields at null infinity on a Schwarzschild background.

\section{Conclusions}

We investigated the asymptotic behavior of spherically symmetric solutions to scalar wave and Yang-Mills equations on a Schwarzschild background. The investigation develops an idea presented in [40] where, among others, decay rates have been used to estimate the validity of the concept of null infinity for predicting detector signals. Our investigation focuses on 
the observer dependence of decay rates. In the case of scalar fields with vanishing angular momentum, a powerful mathematical estimate presented in $[42,43]$ could be compared with a numerical solution. For scalar fields with angular momentum, no such estimate is yet available, but the numerical results suggest that a similar estimate with modified exponents should be valid. For Yang-Mills fields the decay rates near timelike infinity and null infinity have been studied. The breakdown of linear perturbation theory for predicting decay rates near timelike infinity in case of Yang-Mills fields as discovered in [5] could be numerically supported also for decay rates along null infinity.

On the example of the above studies, we emphasized the astrophysical relevance of null infinity. The examples are intended to contribute to the clarification of the question of to what extent null infinity can be regarded as a good idealization for far away observers. The studies also deliver a cautious remark against naive interpretations of finite distance extractions. One needs to be careful in wave extraction not only due to gauge issues in extraction methods and influences from the outer boundary, but also due to the fact that the extraction radius in standard numerical calculations is very small in comparison with astronomical distances. While the tail is certainly a special feature in that respect, there might be other features in the radiation signal that show a non-trivial dependence on the extraction surface. Advanced numerical studies including null infinity should be carried out to clarify this issue as has been initiated within the characteristic approach [24].

The numerical studies of null infinity presented in this paper have been performed by solving hyperboloidal initial value problems [20,23]. This method seems to be quite powerful to study radiative properties of test fields at null infinity once a suitable class of gauges has been chosen $[1,17,30]$. It also promises to be more general than the characteristic approach due to the large freedom involved in the choice of hyperboloidal gauges. In comparison to the standard initial boundary value problem, the numerical implementation is quite simple because one does not need to mathematically construct or numerically implement boundary conditions and data. In addition, the conclusions that can be drawn from the numerical simulation are more powerful as radiative properties of fields can be studied all the way up to null infinity.

In future work, the hyperboloidal initial value problem should be further studied, for example, for different test fields or on different backgrounds such as on Kerr spacetime using a conformal fixing gauge as presented in [46]. The most important challenge in numerical studies of hyperboloidal initial value problems is to devise a successful numerical implementation of the conformally compactified Einstein equations including null infinity. While there are some recent ideas on how to treat a hyperboloidal initial value problem for the Einstein equations directly [1, 30, 34, 45], they have not yet been implemented numerically for interesting cases. Studies of test fields might give further insight into this challenging problem.

\section{Acknowledgments}

I thank Sascha Husa for his help with the calculations on various stages of this research as well as for comments on the manuscript. I would also like to thank Helmut Friedrich, Luciano Rezzolla and Nikodem Szpak for discussions and support.

\section{References}

[1] Andersson L 2002 Lect. Notes Phys. 604183

[2] Barack L 1999 Phys. Rev. D 59044016

[3] Barack L 1999 Phys. Rev. D 59044017

[4] Bartnik R 1992 Relativity Today ed Z Perjes (Commack, USA: Nova Science) pp 221-240 
[5] Bizon P, Chmaj T and Rostworowski A 2007 Class. Quantum Grav. 24 F55

[6] Blanchet L and Sathyaprakash B S 1995 Phys. Rev. Lett. 741067

[7] Bonnor W B and Rotenberg M A 1966 Proc. R. Soc. Lond. A 289247

[8] Boyle M et al 2007 Phys. Rev. D 76124038

[9] Brill D R, Cavallo J M and Isenberg J A 1980 J. Math. Phys. 212789

[10] Burko L M and Ori A 1997 Phys. Rev. D 567820

[11] Cai R-G and Wang A 1999 Gen. Rel. Grav. 311367

[12] Campanelli M, Gomez R, Husa S, Winicour J and Zlochower Y 2001 Phys. Rev. D 63124013

[13] Ching E S C, Leung P T, Suen W M and Young K 1995 Phys. Rev. D 522118

[14] Choptuik M W, Chmaj T and Bizoń P 1996 Phys. Rev. Lett. 77424

[15] Dafermos M 2004 Preprint gr-qc/0401121

[16] Dafermos M and Rodnianski I 2005 Invent. Math. 162381

[17] Frauendiener J 1998 Phys. Rev. D 58064003

[18] Frauendiener J 2000 Class. Quantum Grav. 17373

[19] Frauendiener J 2004 Living Rev. Rel. 7 (http://www.livingreviews.org/lrr-2004-1)

[20] Friedrich H 1983 Commun. Math. Phys. 91445

[21] Friedrich H 2002 Lect. Notes Phys. 6041

[22] Gentle A P, Holz D E, Kheyfets A, Laguna P, Miller W A and Shoemaker D M 2001 Phys. Rev. D 63064024

[23] Geroch R 1977 Asymptotic Structure of Space-Time ed F Esposito and L Witten (New York: Plenum) pp 1-105

[24] Gómez R and Winicour J 1992 Phys. Rev. D 452776

[25] Gundlach C, Price R H and Pullin J 1994a Phys. Rev. D 49883

[26] Gundlach C, Price R H and Pullin J 1994b Phys. Rev. D 49890

[27] Hannam M, Husa S, Sperhake U, Brügmann B and Gonzalez J A 2008 Phys. Rev. D 77044020

[28] Huebner P 1996 Phys. Rev. D 53701

[29] Husa S 2003 Current Trends in Relativistic Astrophysics (Lecture Notes in Physics vol 617) ed L Fernández and L M González (Berlin: Springer) (Preprint gr-qc/0204057)

[30] Husa S, Schneemann C, Vogel T and Zenginoğlu A 2006 AIP Conf. Proc. 841306

[31] Husa S, Zlochower Y, Gomez R and Winicour J 2002 Phys. Rev. D 65084034

[32] Leaver E W 1986 Phys. Rev. D 34384

[33] Malec E and Murchadha N O 2003 Phys. Rev. D 68124019

[34] Moncrief V 2006 The conformally reduced Einstein equations-an alternative approach to SCRI Talk at IHP (Paris)

[35] Pazos E, Dorband E N, Nagar A, Palenzuela C, Schnetter E and Tiglio M 2007 Class. Quantum Grav. 24 S341

[36] Penrose R 1963 Phys. Rev. Lett. 1066

[37] Penrose R 1964 Relativity, Groups and Topology ed C DeWitt and B DeWitt (New York: Gordon and Breach) pp 565-84

[38] Penrose R 1965 Proc. R. Soc. Lond. A 284159

[39] Price R 1972 Phys. Rev. D 52419

[40] Pürrer M, Husa S and Aichelburg P C 2005 Phys. Rev. D 71104005

[41] Rinne O, Lindblom L and Scheel M A 2007 Class. Quantum Grav. 244053

[42] Szpak N 2007 Preprint arXiv:0710.1782

[43] Szpak N, Bizon P, Chmaj T and Rostworowski A 2007 Preprint arXiv:0712.0493

[44] Winicour J 2005 Living Rev. Rel. 810 (http://www.livingreviews.org/lrr-2005-10)

[45] Zenginoğlu A 2007 A conformal approach to numerical calculations of asymptotically flat spacetimes $P h D$ Thesis University of Potsdam (Preprint arXiv:0711.0873)

[46] Zenginoğlu A 2008 Class. Quantum Grav. 25145002 